\title{
ESeroS-GS Protects Neuronal Cells from Oxidative Stress by Stabilizing Lysosomes
}

\author{
Na Yang ${ }^{1,2}$, Qianqian Chen ${ }^{1,2}$, Xiaolong $\mathrm{He}^{1,2}$, Xingyu Zhao ${ }^{1}$ and Taotao Wei ${ }^{1, *}$ \\ 1 National Laboratory of Biomacromolecules, Institute of Biophysics, Chinese Academy of Sciences, \\ Beijing 100101, China; nayang2007@126.com (N.Y.); chenqian0621@163.com (Q.C.); \\ xiaolonghe@yeah.net (X.H.); pkuibpzxy@gmail.com (X.Z.) \\ 2 University of Chinese Academy of Sciences, Beijing 100049, China \\ * Correspondence: weitt@moon.ibp.ac.cn; Tel.: +86-10-6488-8566
}

Academic Editor: Maurizio Battino

Received: 24 March 2016; Accepted: 10 May 2016; Published: 25 May 2016

\begin{abstract}
L-glutamyl-S-[2-[[[3,4-dihydro-2,5,7,8-tetramethyl-2-(4,8,12-trimethyltridecyl)-2H-1benzopyran-6-yl]oxy]carbonyl]-3-[[2-(1H-indol-3-yl)ethyl]amino]-3-oxopropyl]-L-cysteinylglycine sodium salt (ESeroS-GS) is a water-soluble derivative of $\alpha$-tocopherol (vitamin E). We reported previously that ESeroS-GS can act as an anti-inflammatory agent and can induce cell death in breast cancer cells. However, the potential antioxidant capacities of ESeroS-GS remain elusive. Here, we measured its scavenging effects on free radicals and evaluated its protective effects on neuronal cells against oxidative stress. The results indicated that ESeroS-GS effectively scavenged both 2,2'-azinobis(3-ethylbenzothiazoline)-6-sulfonate free radicals (ABTS ${ }^{\bullet+}$ ) and 2,2-diphenyl-1-picrylhydrazyl (DPPH) free radicals, and attenuated $\mathrm{H}_{2} \mathrm{O}_{2}$-induced neuronal cell death. $\mathrm{H}_{2} \mathrm{O}_{2}$ treatment induced lysosomal membrane permeabilization rapidly, and caused the redistribution of lysosomal proteases, which were responsible for the neuronal cell death. ESeroS-GS abolished the interaction between $\mathrm{tBid}$ and the lysosomal membranes, blocked the translocation of tBid to the lysosomal membranes, decreased its oligomerization within the membrane circumstances, prevented the lysosomal membrane permeabilization, and thus attenuated the neuronal cell death. These data suggest that ESeroS-GS protected the neuronal cells from oxidative stress by stabilizing lysosomal membranes, and thus might act as a novel neuroprotector for neuronal diseases associated with oxidative stress.
\end{abstract}

Keywords: ESeroS-GS; antioxidant; neuronal cell; cell death; oxidative stress; lysosome; Bid

\section{Introduction}

In our body, a number of biochemical reactions involve the generation of reactive oxygen species (ROS). The major sources of endogenous ROS are mitochondria. Leakage of electrons during oxidative phosphorylation causes, depending on the animal species and rate of respiration, the conversion of $0.25 \%-11 \%$ of the oxygen utilized to superoxide anion radicals and, ultimately, $\mathrm{H}_{2} \mathrm{O}_{2}$ [1]. Under certain pathological conditions, when ROS are not effectively eliminated by the antioxidant defense system, the dynamic balance between the generation and diminution of ROS is broken. Excessive ROS can attack lipids, carbohydrates, proteins and DNA, thus resulting in oxidative stress. Several lines of evidence link ROS to the onset of a variety of pathologic events, such as coronary heart disease [2], cancer [3], type 2 diabetes [4], and the degenerative diseases associated with aging [5,6].

One of the hallmarks of neurodegeneration is the loss of certain neurons. ROS can contribute to neuronal cell death by either apoptosis or necrosis, and mitochondria are viewed as one of the most pivotal sensors and amplifiers of cell death [7]. Mitochondrial outer membrane permeabilization results in the release of multiple proteins, such as cytochrome $c$ and endonuclease $G$, into the cytosol. 
This leads to caspase activation in the cytosol, disruption of the mitochondrial respiratory chain, loss of mitochondrial transmembrane potential, overproduction of ROS, finally resulting in cell death [8-10].

Recently accumulating evidence has indicated that, in addition to mitochondria, lysosomes also play important roles in the cell death signaling induced by ROS [11-13]. The initiation of lysosomal membrane permeabilization (LMP) is one of the crucial steps leading to cell death. As a result of the degradation of iron-containing macromolecules, lysosomes can accumulate large amounts of iron, which is known to catalyze Fenton reactions. High levels of ROS attack the membrane lipids, damage the integrity of lysosomal membranes directly, resulting in the release of proteases into the cytosol and the induction of uncontrolled necrotic cell death; in contrast, mild oxidative stress initiates a cascade of events leading to the formation of "pores" on lysosomal membrane, causing partial and selective LMP and inducing apoptotic cell death. Multitudes of parallel pathways involved in ROS-induced LMP make cell death a highly complex process. An important molecule that bridges the LMP and cell death is Bid (BH3 interacting domain death agonist) [14]. Our previous findings indicated that, during the initiation phase of ROS-induced cell death, tBid (truncated Bid) formed by activated caspase 8 is targeted and inserted into the lysosomal membranes by interaction with phosphatidic acid, where tBid changes conformation, forms homooligomers, and triggers the formation of non-bilayer lipid phases, which account for the formation of lipidic pores and the consequent initiation of LMP. As a result of LMP, lysosomal proteases are redistributed into the cytosol, where they culminate in the lysosome-dependent apoptotic signaling [15]. Accordingly, agents that can stabilize the lysosomal membranes might protect the cells from lysosome-dependent cell death.

Various molecules derived from natural antioxidant vitamins or micronutrients show neuroprotection effects in vitro and in vivo, probably by attenuating ROS-mediated cell death [16,17]; however, whether they prevent cell death by stabilizing lysosomes, and, if yes, what the underlying mechanisms are remain elusive. In this study, we investigated the effects of a vitamin E derivative, $\gamma$-L-glutamyl-S-[2-[[[3,4-dihydro-2,5,7,8-tetramethyl-2-(4,8,12-trimethyltridecyl)-2H-1-benzopyran-6 -yl]oxy]carbonyl]-3-[[2-(1H-indol-3-yl)ethyl]amino]-3-oxopropyl]-L-cysteinylglycine sodium salt (ESeroS-GS), on ROS-induced neuronal cell death. The molecular mechanisms by which ESeroS-GS stabilizes lysosomes were also explored.

\section{Results and Discussion}

\subsection{ESeroS-GS Scavenged $A B T S^{\bullet+}$ and DPPH Free Radicals}

$\gamma$-L-glutamyl-S-[2-[[[3,4-dihydro-2,5,7,8-tetramethyl-2-(4,8,12-trimethyltridecyl)-2H-1-benzopyran-6-yl]oxy]carbonyl]-3-[[2-(1H-indol-3-yl)ethyl]amino]-3-oxopropyl]-L-cysteinylglycine sodium salt (ESeroS-GS; Figure 1A) is a water-soluble derivative of $\alpha$-tocopherol (vitamin E). We reported previously that ESeroS-GS can act as an anti-inflammatory agent that inhibits the activation of astrocytes [18] or macrophages [19] by modulating NF-kB signaling via a lipid raft-dependent mechanism. We also found that ESeroS-GS induced cell death in different breast cancer cells but showed no significant effects on MCF-10A mammary epithelial cells [20]. However, the potential antioxidant properties of ESeroS-GS remain elusive. Taking the multifactorial character of oxidative stress into account, we decided to evaluate its in vitro free radical scavenging abilities using two different assays.

Firstly, we determined the scavenging activities of ESeroS-GS against the hydrophilic cation radical of 2,2'-azinobis(3-ethylbenzothiazoline)-6-sulfonate $\left(\mathrm{ABTS}^{\bullet+}\right)$ by measuring the decolorization of the $\mathrm{ABTS}^{\bullet+}$ radicals at $734 \mathrm{~nm}$ [21,22]. 6-Hydroxy-2,5,7,8-tetramethy-chroman-2-carboxylic acid (Trolox), a water-soluble analog of $\alpha$-tocopherol, was used as the reference compound. The extent of scavenging of the $\mathrm{ABTS}^{\bullet+}$ was plotted as a function of antioxidant concentration, as shown in Figure 1B. Both ESeroS-GS and Trolox scavenged ABTS ${ }^{\bullet+}$ free radicals dose-dependently. The $\mathrm{IC}_{50}$ values for ESeroS-GS and Trolox in scavenging ABTS $^{\bullet+}$ radicals were 31.4 and $13.5 \mu \mathrm{M}$, respectively. 

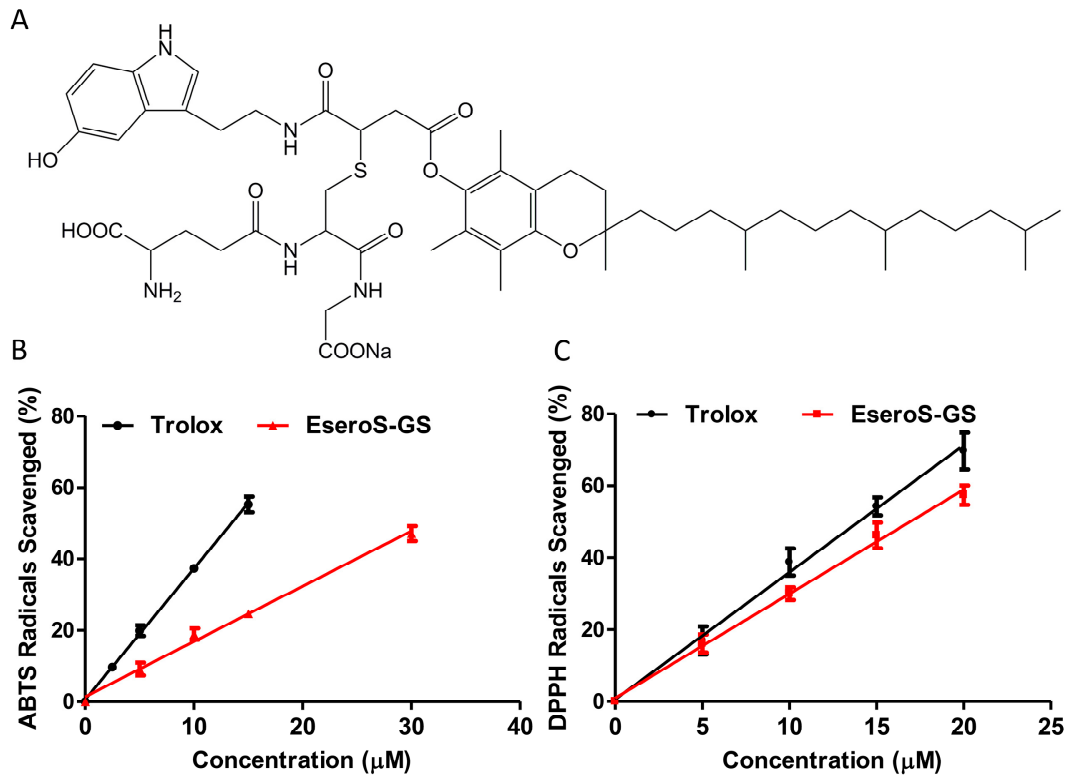

Figure 1. Scavenging effects of ESeroS-GS and Trolox on free radicals. (A) structure of ESeroS-GS; (B) scavenging effects of ESeroS-GS and Trolox on ABTS ${ }^{\bullet+}$ free radicals; (C) scavenging effects of ESeroS-GS and Trolox on DPPH free radicals.

Then, we determined the scavenging activities of ESeroS-GS and Trolox against hydrophobic 2,2-diphenyl-1-picrylhydrazyl (DPPH) stable free radicals [23,24]. ESeroS-GS decreased the signal of DPPH radicals in a concentration-dependent manner, as shown in Figure $1 \mathrm{C}$. The $\mathrm{IC}_{50}$ values for ESeroS-GS and Trolox in scavenging DPPH radicals were 16.9 and $14.3 \mu \mathrm{M}$, respectively.

\subsection{ESeroS-GS Protected Neuronal Cells from Oxidative Stress}

Since both ESeroS-GS and Trolox scavenged free radicals effectively, we then tested their potential protect effects on neuronal cells exposed to oxidative stress. Primary cultures of cerebellar granule cells, a relatively homogenous population of neurons, were used as the cell model. The viability of neuronal cells was assessed by the 3-(4,5-dimethyl-2-thiazolyl)-2,5-diphenyl-2-H-tetrazolium bromide (MTT) assay, which is based on the reduction of MTT by mitochondrial dehydrogenases. As shown in Figure $2 \mathrm{~A}$, treatment with $100 \mu \mathrm{M} \mathrm{H}_{2} \mathrm{O}_{2}$ decreased the cell viability to $72.5 \%$. In cerebellar granule cells pretreated with $50 \mu \mathrm{M}$ of ESeroS-GS, treatment with $100 \mu \mathrm{M} \mathrm{H}_{2} \mathrm{O}_{2}$ decreased the cell viability to $86.3 \%$, suggesting that ESeroS-GS attenuated $\mathrm{H}_{2} \mathrm{O}_{2}$-induced cell death effectively. However, Trolox did not show apparent protective effects on neuronal cells, even at higher concentrations $(100 \mu \mathrm{M})$.
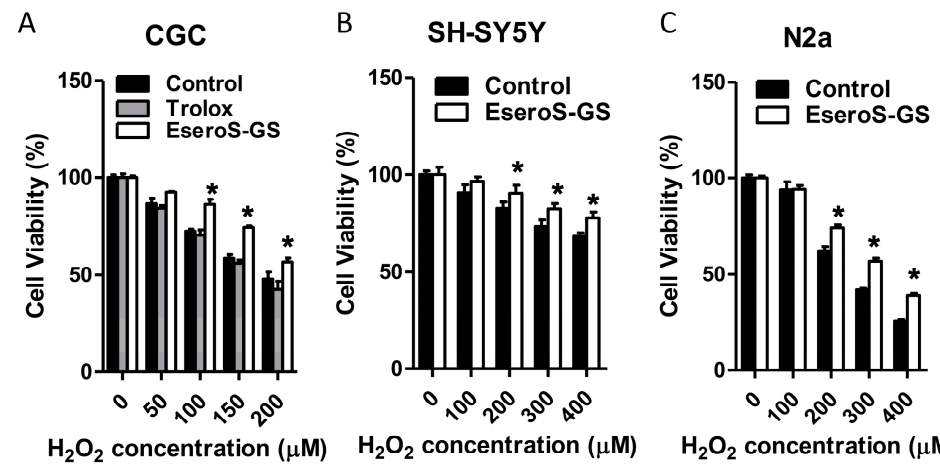

Figure 2. Protective effects of ESeroS-GS on neuronal cells. (A) protective effects of ESeroS-GS on cerebellar granule cells; (B) protective effects of ESeroS-GS on human SH-SY5Y neuroblastoma cells; (C) protective effects of ESeroS-GS on murine N2a neuroblastoma cells. ${ }^{*}: p<0.05$ in comparison with control cells. 
The protective effects of ESeroS-GS against oxidative stress-induced cell death were further evaluated with two immortalized cell lines, the human SH-SY5Y and the murine N2a neuroblastoma cells. ESeroS-GS pretreatment also attenuated $\mathrm{H}_{2} \mathrm{O}_{2}$-induced cell death in these two cell lines, as shown in Figure 2B,C.

\subsection{Relocation of Lysosomal Chymotrypsin Mediated Cell Death}

Although Trolox is more effective than ESeroS-GS in scavenging ABTS ${ }^{\bullet+}$ and DPPH free radicals, it showed no apparent protective effects on $\mathrm{H}_{2} \mathrm{O}_{2}$-induced neuronal cell death. We then hypothesized that ESeroS-GS might protect the neuronal cells not only by scavenging radicals directly, but also via other mechanisms. Lysosomes are one of the pivotal targets of oxidative stress within cells [13,25]. To investigate whether lysosomes are involved in $\mathrm{H}_{2} \mathrm{O}_{2}$-induced neuronal cell death, we measured the permeability of lysosomal membranes after $\mathrm{H}_{2} \mathrm{O}_{2}$ exposure by using acridine orange (AO) as the lysosomotropic fluorophore. $\mathrm{AO}$ is a cell-permeable dye that gives rise to red fluorescence at high concentrations and green fluorescence at low concentrations. $\mathrm{AO}$ accumulates by proton trapping in intact lysosomes due to the fact that it becomes positively charged in the acidic lysosomal milieu. Following lysosomal membrane permeabilization, $\mathrm{AO}$ is released from lysosomes into the cytosol where it emits enhanced green fluorescence that can be monitored by flow cytometry [15].

The results of flow cytometry indicated that, as a result of oxidative stress, over $40 \%$ of SH-SY5Y cells underwent lysosomal membrane permeabilization, as evidenced by the decreased red fluorescence (lysosomal AO) and the increased green fluorescence (cytosolic AO). Pretreatment with ESeroS-GS attenuated the permeabilization of lysosomal membrane effectively in a concentration-dependent manner. In SH-SY5Y cells pretreated with $50 \mu \mathrm{M}$ of ESeroS-GS, the percentage of cells with permeabilized lysosomes decreased to $14.1 \%$ (Figure 3A).
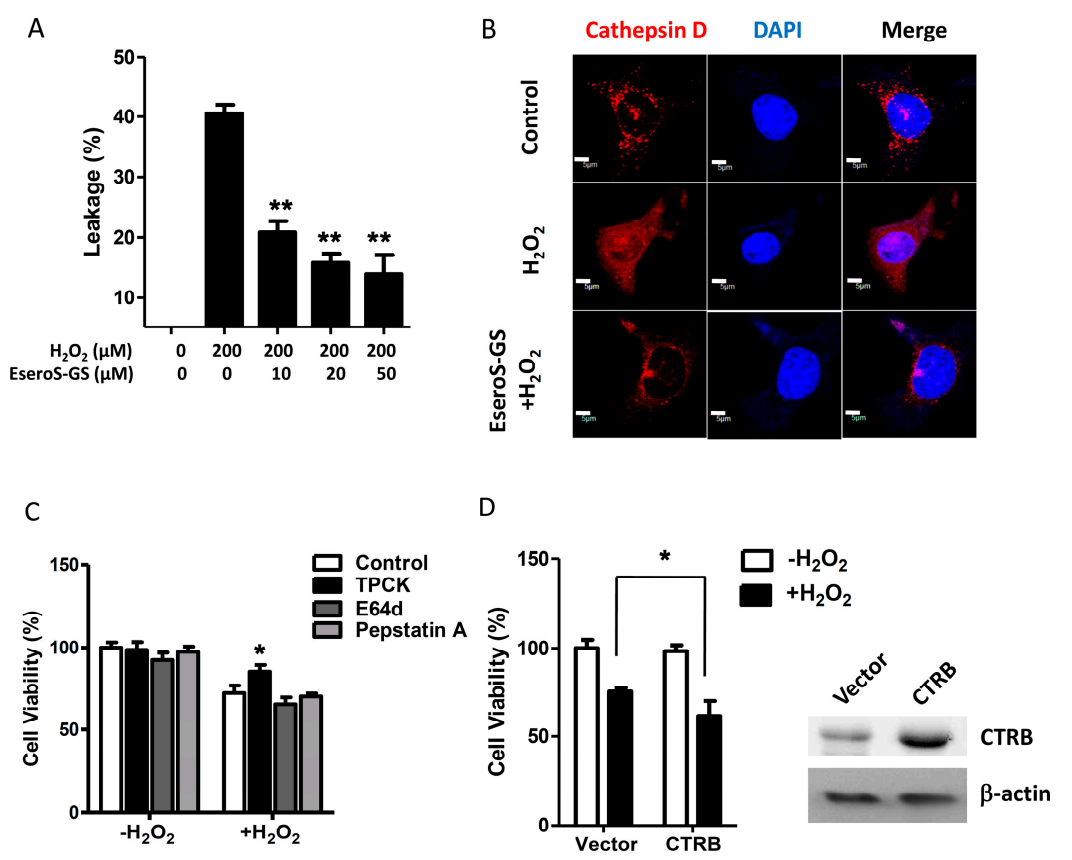

Figure 3. ESeroS-GS inhibits oxidative stress-induced lysosome membrane permeabilization and blocks the relocation of lysosomal chymotrypsin in SH-SY5Y neuroblastoma cells. (A) ESeroS-GS inhibits oxidative stress-induced lysosome membrane permeabilization; (B) ESeroS-GS blocks the relocation of lysosomal protease. Scale bar: $5 \mu \mathrm{m}$; (C) inhibition of chymotrypsin prevents oxidative stress-induced cell death; (D) Overexpression of chymotrypsin potentiates oxidative stress-induced cell death. The efficacy of chymotrypsin overexpression was evaluated by immunoblot analysis. Transfection with chymotrypsin B (CTRB) vector resulted in a marked ( $\sim 5$ times) increase in cellular chymotrypsin levels in SH-SY5Y neuroblastoma cells. ${ }^{*}: p<0.05 ; * *: p<0.01$. 
To further confirm that oxidative stress damaged the lysosomal integrity and triggered the redistribution of lysosomal proteases, we observed the translocation of lysosomal protease cathepsin $\mathrm{D}$, a marker lysosomal protease, in oxidative stressed SH-SY5Y cells by immunofluorescence microscopy. In control cells, cathepsin D (red) was cached in the lysosomes and showed a punctuate pattern (Figure 3B). Exposure of cells to oxidative stress caused the redistribution of cathepsin D into the cytosol, where they showed a diffused pattern. With the ESeroS-GS pretreatment, the redistribution of cathepsin D into the cytosol was attenuated markedly, suggesting that ESeroS-GS prevented the lysosomal membrane from permeabilization and blocked the relocation of lysosomal proteases.

Upon lysosomal membrane permeabilization, dozens of lysosomal proteases were relocated into the cytosol, where they cleaved target molecules and executed the lysosome-dependent cell death. To ascertain which lysosomal protease(s) was (were) responsible for the oxidative stress-cell death, we pretreated SH-SY5Y cells with different protease inhibitors. The results shown in Figure 3C indicated that $N$-p-tosyl-L-phenylalanine chloromethyl ketone (TPCK), the specific chymotrypsin inhibitor, attenuated $\mathrm{H}_{2} \mathrm{O}_{2}$-induced cell death in SH-SY5Y cells. However, $(2 S, 3 S)$-trans-epoxysuccinyl-L-leucylamido-3-methylbutane ethyl ester (E-64d) and pepstatin A, two inhibitors of the lysosomal cathepsin families, had no apparent effect on cell death.

We further manipulated the expression levels of chymotrypsin to ascertain the involvement of chymotrypsin in cell death. Overexpression of chymotrypsin sensitized SH-SY5Y cells to oxidative stress, as shown in Figure 3D. Upon $\mathrm{H}_{2} \mathrm{O}_{2}$ exposure, the viability of SH-SY5Y transfected with chymotrypsin expression vector was $61.5 \%$, significantly lower than that of control cells. These data were consistent with the partial reduction of $\mathrm{H}_{2} \mathrm{O}_{2}$-induced cell death by the chymotrypsin inhibitor TPCK, and clearly indicated that lysosomal chymotrypsin play crucial roles in $\mathrm{H}_{2} \mathrm{O}_{2}$-induced neuronal cell death.

\subsection{ESeroS-GS Stabilized Lysosomal Membranes}

The above data suggested that pretreating neuronal cells with ESeroS-GS effectively decreased the permeabilization of lysosomal membranes, attenuated the redistribution of lysosomal proteases into the cytosol, and prevented neuronal cells from cell death. We then asked by which mechanisms ESeroS-GS stabilize the lysosomal membranes. Our previous data indicated that tBid is responsible for the induction of lysosomal membrane permeabilization during cell death [15]. We then observed the effects of ESeroS-GS on tBid-mediated membrane permeabilization in a relatively simple context by using isolated lysosomes and liposomes that mimick the lipid composition of lysosomes.

We first measured the effects of ESeroS-GS on tBid-mediated permeabilization of model membranes (large unilamellar vesicles; LUVs). As shown in Figure 4A, tBid induced the leakage of LUVs, mimicking the phospholipid composition of lysosomal membranes significantly. In LUVs incubated with tBid, up to $47.5 \%$ of the contents encapsulated within the LUVs were released as a result of membrane permeabilization. The addition of ESeroS-GS attenuated the tBid-induced membrane permeabilization effectively, and only $16.8 \%$ of the LUV contents were released.

Our previous data indicated that the translocation of tBid to lysosomes is the initial key step in the induction of lysosomal membrane permeabilization; we then measured the impact of ESeroS-GS on tBid translocation to lysosomes in a cell-free system. Lysosomes were isolated from rat cerebral cortex and then were incubated with tBid. As shown in Figure 4B, incubation of tBid with lysosomes caused its significant translocation to the lysosomal membranes, and the addition of ESeroS-GS could decrease translocation of tBid to the lysosomal membranes.

The impact of ESeroS-GS on tBid translocation was also investigated with model membrane systems. tBid translocated to the phosphatidic acid-containing LUVs rapidly, as shown in Figure 4B, and this translocation could be partially abolished by ESeroS-GS, similar to the results observed in isolated lysosomes. 
A

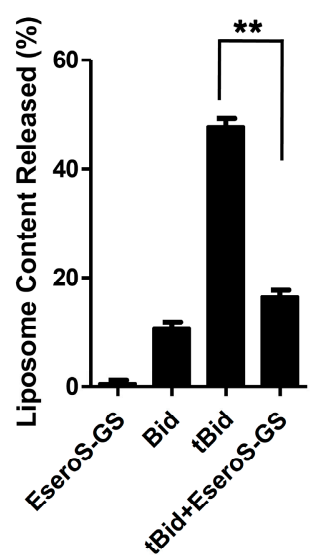

C

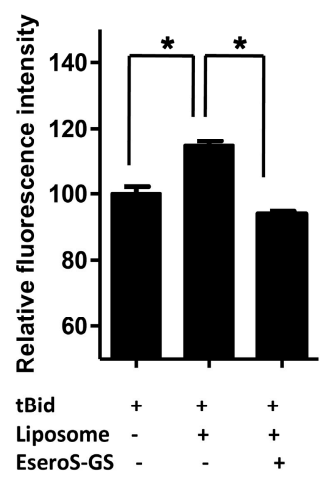

B
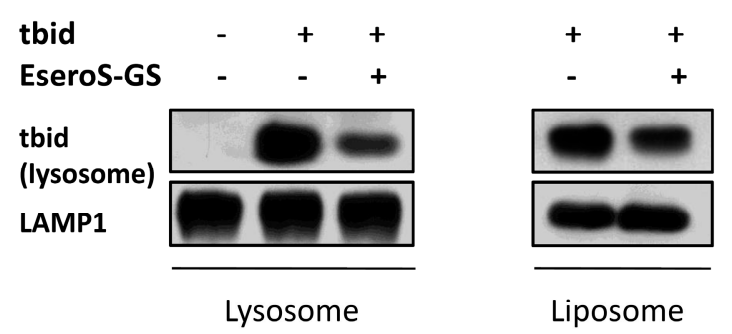

tbid

(liposome)

tbid

(input)

D

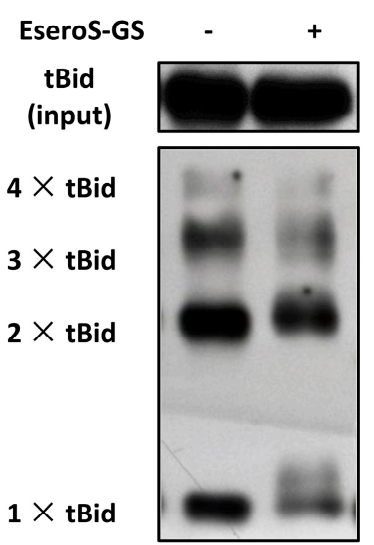

Figure 4. ESeroS-GS stabilizes lysosomal membranes by inhibiting the insertion and oligomerization of tBid to membranes. (A) ESeroS-GS inhibits tBid-induced permeabilization of model membranes; (B) ESeroS-GS attenuates the translocation of tBid to lysosomes and model membranes; (C) ESeroS-GS decreases the interaction between $\mathrm{tBid}$ and model membranes; (D) ESeroS-GS blocks the oligomerization of tBid within the membrane circumstances. * $p<0.05 ;{ }^{* *}: p<0.01$.

We also determined the effects of ESeroS-GS on the interaction between tBid and lipid bilayer by fluorescence spectrometry. $\mathrm{N}$-(1-pyrenyl)maleimide (PM) is an environmentally sensitive fluorescent probe, whose fluorescence intensity increases obviously in a hydrophobic environment. The fluorescence intensity of tBid labeled with PM was increased after incubating with liposomes, suggesting the insertion of tBid into the lipid bilayer. However, the addition of ESeroS-GS decreased the PM fluorescence intensity significantly, suggesting that the insertion of tBid into the lipid bilayer was blocked by ESeroS-GS (Figure 4C).

After the insertion of tBid molecules into the membrane, they formed homooligomers, which were the structural basis of the lipidic pore that was responsible for the membrane permeabilization. We further investigated the effects of ESeroS-GS on tBid oligomerization in model membranes, mimicking the phospholipid composition of lysosomal membranes. tBid was incubated with liposomes followed by centrifugation to separate the membranous fraction from the soluble fraction. Both fractions were treated with the bis[2-(sulfosuccinimidooxycarbonyloxy)ethyl] sulfone (sulfo-BSOCOES) cross-linker and analyzed by immunoblotting. After adding tBid to liposomes, a substantial amount of $\mathrm{tBid}$ was incorporated into the liposomal membrane, with the appearance of $14-\mathrm{kDa}$ (monomer), $28-\mathrm{kDa}$ (dimer), $42-\mathrm{kDa}$ (trimer), $56-\mathrm{kDa}$ (tetramer) and higher molecular weight band (homooligomers) cross-linked complexes. Significantly less tBid oligomerization was incorporated into the liposomal membrane when EseroS-GS was added into the liposomes, suggesting that ESeroS-GS attenuated the oligomerization of tBid. 


\section{Materials and Methods}

\subsection{Chemicals and Antibodies}

$\gamma$-L-Glutamyl-S-[2-[[[3,4-dihydro-2,5,7,8-tetramethyl-2-(4,8,12-trimethyltridecyl)-2H-1-benzo-pyran6-yl]oxy]carbonyl]-3-[[2-(1H-indol-3-yl)ethyl]amino]-3-oxopropyl]-L-cysteinylglycine sodium salt (ESeroS-GS) was a generous gift from Kazumi Ogata of Senju Pharmaceutical Co. Ltd. (Osaka, Japan). 6-Hydroxy-2,5,7,8-tetramethychroman-2-carboxylic acid (Trolox), 2,2'-azinobis (3-ethylbenzothiozoline6-sulphonic acid) diammonium salt (ABTS), 1,1-dipheny-2-picrylhydrazyl (DPPH), 5-carboxyfluorescein (CF), N-p-tosyl-L-phenylalanine chloromethyl ketone (TPCK), $(2 S, 3 S)$-trans-epoxysuccinyl-L-leucylamido3-methylbutane ethyl ester (E-64d), pepstatin A, 2-[4-(2-Hydroxyethyl)-1-piperazinyl]ethanesulfonic acid (HEPES), 3-(4,5-dimethyl-2-thiazolyl)-2,5-diphenyl-2-H-tetrazolium bromide (MTT) and ethylenediaminetetraacetic acid disodium salt (EDTA) were from Sigma-Aldrich (St Louis, MO, USA). 1,2-Dioleoyl-sn-glycero-3-phosphate (DOPA), 1,2-dioleoyl-sn-glycero-3-phosphocholine (DOPC) and 1,2-dioleoyl-sn-glycero-3-phosphoethanolamine (DOPE) were from Avanti Polar Lipids (Alabaster, AL, USA). Acridine orange (AO), N-(1-pyrenyl)maleimide (PM), 4',6-diamidino-2-phenylindole (DAPI), cell culture medium, cell culture supplements and fetal bovine serum were from Life Technologies (Eugene, OR, USA). Primary antibodies against cathepsin D and Bid were from Santa Cruz Biotechnology (Santa Cruz, CA, USA). Horseradish peroxidase (HRP)-labeled and 6-tetramethylrhodamine isothiocyanate (TRITC)-labeled second antibodies were from Sigma-Aldrich. Other reagents made in China were of analytical grade.

\subsection{Expression and Labeling of Recombinant Proteins}

Recombinant full-length Bid and caspase 8 were obtained as we described previously [26]. Truncated Bid (tBid; includes N-terminal fragment 1-59 and C-terminal fragment 60-195) was obtained by adding caspase 8 to the full-length $\mathrm{Bid}$, and then incubating overnight at $37{ }^{\circ} \mathrm{C}$ [27]. In some experiments, tBid was labeled with fluorescent probe $\mathrm{N}$-(1-pyremyl)maleimide (PM). Before labeling, Bid was dialyzed extensively against Tris buffer $(20 \mathrm{mM}$ Tris- $\mathrm{HCl}, \mathrm{pH} 7.5,300 \mathrm{mM} \mathrm{NaCl})$ and then labeled with PM dye ( $3 \mathrm{~mol}$ of PM to $1 \mathrm{~mol}$ of $\mathrm{tBid}$ ) in the same buffer with rotation at $30^{\circ} \mathrm{C}$ for $2 \mathrm{~h}$ in the dark. After the reaction, the protein sample was dialyzed against Tris buffer to remove the excessive PM dye.

\subsection{Free Radical Scavenging Assays}

The scavenging effect of ESeroS-GS on the hydrophilic ABTS free radicals $\left(\mathrm{ABTS}^{\bullet+}\right)$ was measured by the decolorization of $\mathrm{ABTS}^{\bullet+}$ at $734 \mathrm{~nm}[21,22]$. ABTS dissolved in water at a $7 \mathrm{mM}$ concentration was reacted with $2.45 \mathrm{mM}$ potassium persulfate in the dark at room temperature for $12-16 \mathrm{~h}$. Then, the ABTS $^{\bullet+}$ solution was diluted with $150 \mathrm{mM}$ phosphate buffer (pH 7.3) to an absorbance of $0.700 \pm 0.020$ at $734 \mathrm{~nm}$ and equilibrated at $30^{\circ} \mathrm{C}$. After addition of $1.0 \mathrm{ml}$ of diluted ABTS ${ }^{\bullet+}$ solution $\left(\mathrm{A}_{734 \mathrm{~nm}}=0.700 \pm 0.020\right)$ to $10 \mu \mathrm{L}$ of ESeroS-GS or Trolox solutions (final concentration $0 \sim 15 \mu \mathrm{M}$ ), the absorbance reading at $734 \mathrm{~nm}$ was taken exactly 5 min after initial mixing. The percentage scavenging of $\mathrm{ABTS}^{\bullet+}$ was calculated by:

$$
\mathrm{I}(\%)=[(\mathrm{Ao}-\mathrm{Ax}) / \mathrm{Ao}] \times 100
$$

Here, Ax and Ao were the absorbance at $734 \mathrm{~nm}$ of samples with and without antioxidants, respectively.

The scavenging of hydrophobic DPPH free radicals by ESeroS-GS was examined by the Electron Spin Resonance (ESR) technique [23,24]. DPPH was dissolved in ethanol to give a $100 \mu \mathrm{M}$ solution and mixed with equal volumes of ESeroS-GS or Trolox solutions, then transferred to quartz capillary which was inserted into the cavity of the Bruker ER200SRC ESR spectrometer (Bruker, Karlsruhe, Germany). ESR spectra were recorded exactly 2 min after initial mixing. ESR measurement conditions were as follows: central field $3475 \mathrm{G}$, modulation frequency $100 \mathrm{kHz}$, modulation amplitude 2 G, microwave 
power $10 \mathrm{~mW}$, scan width $200 \mathrm{G}$, gain $6.3 \times 10^{5}$, temperature $298 \mathrm{~K}$. The scavenging effects on DPPH free radical were calculated by:

$$
\mathrm{I}(\%)=[(\mathrm{Ho}-\mathrm{Hx}) / \mathrm{Ho}] \times 100
$$

Here, Hx and Ho were the ESR signal intensities of samples with and without antioxidants, respectively.

\subsection{Cell Culture and $\mathrm{H}_{2} \mathrm{O}_{2}$ Treatment}

Primary cultures of rat cerebellar granule cells were prepared from postnatal day 6Sprague-Dawley rat pups (Vital River Experimental Animal Center, Beijing, China), with the regulations approved by the Institutional Animal Care and Use Committee of Institute of Biophysics, Chinese Academy of Sciences (IACUC-IBP) [28]. Cells were plated on 96-well multidishes previously coated with poly-L-lysine. Culture medium consisted of Dulbecco's modified Eagle medium (DMEM) supplemented with $\mathrm{KCl}(19.6 \mathrm{mM})$, glutamine $(2 \mathrm{mM})$, HEPES $(10 \mathrm{mM})$ and fetal bovine serum $(10 \%, v / v)$. Mouse neuroblastoma N2a cells and human neuroblastoma SH-SY5Y cells were obtained from the American Type Culture Collection (ATCC; Manassas, VA, USA) and were cultured under conditions recommended by ATCC. Cerebellar granule cells, N2a cells and SH-SY5Y cells were exposed to oxidative stress by incubation with $\mathrm{H}_{2} \mathrm{O}_{2}$ in serum-free cell culture medium for 30 min and then cultured in fresh medium containing serum for $12 \mathrm{~h}$. In some experiments, cells were pretreated with ESeroS-GS, Trolox, TPCK, E-64d or pepstatin for $1 \mathrm{~h}$ before $\mathrm{H}_{2} \mathrm{O}_{2}$ exposure.

\subsection{Transient Overexpression of Chymotrypsin}

For the construction of chymotrypsin overexpression vectors, open reading frames encoding chymotrypsinogen were amplified by PCR with appropriate restriction sites and cloned into pcDNA3.1 vector, producing a pcDNA3.1-Ctr vector encoding myc-tagged chymotrypsin [26]. SH-SY5Y cells were transfected with pcDNA3.1-Ctr vector by using Fugene HD reagent (Promega, Madison, WI, USA) according to the manufacturer's instructions. Then, cells were exposed to $200 \mu \mathrm{M} \mathrm{H}_{2} \mathrm{O}_{2}$ for $30 \mathrm{~min}$ and cultured in fresh medium for $12 \mathrm{~h}$. Immunoblot analysis was performed to evaluate the efficacy of chymotrypsin overexpression.

\subsection{Assessment of Cell Death}

The viability of cells was evaluated by MTT assay, according to methods we described previously [20]. In brief, cells cultured in 96-well multi-dishes were treated with different concentration of $\mathrm{H}_{2} \mathrm{O}_{2}$ for $30 \mathrm{~min}$, and then cultured in fresh medium for $12 \mathrm{~h}$. MTT reagent was added into cells and cells were incubated for additional $1 \mathrm{~h}$. Then, $0.2 \mathrm{~mL}$ of lysis solution (10\% SDS, 25\% $\mathrm{N}, \mathrm{N}$-dimethylformamide, $\mathrm{pH} 3.5$ ) was added and the optical density at $570 \mathrm{~nm}$ was measured by a Thermo Fisher microplate reader (Thermo Fisher, Beijing, China).

\subsection{Measurement of Lysosomal Membrane Permeabilization}

Lysosomal permeabilization was determined in intact cells by staining with acridine orange (AO), a lysosomotropic fluorophore [29,30]. In brief, SH-SY5Y cells were loaded with AO $(5 \mu \mathrm{g} / \mathrm{mL})$ for $30 \mathrm{~min}$ and then exposed to $200 \mu \mathrm{M} \mathrm{H}_{2} \mathrm{O}_{2}$ for $30 \mathrm{~min}$. AO-emitted red (lysosomal) and green (nuclear and cytosolic) fluorescence were analyzed with a flow cytometer (FACSCalibur; BD, Mountain View, CA, USA). Cells with enhanced green fluorescence and decreased red fluorescence were regarded as lysosomal membrane-permeabilized cells.

The permeabilization of lysosomes was further confirmed by the relocation of lysosomal cathepsin D. SH-SY5Y cells cultured on glass coverslips were fixed with cold methanol in $-20^{\circ} \mathrm{C}$ for $20 \mathrm{~min}$ and incubated with ice-cold solution containing 95\% ethyl alcohol and 1\% acetic acid for $5 \mathrm{~min}$. After washing with PBS twice, cells were blocked for $1 \mathrm{~h}$ at room temperature with PBS 
containing 5\% goat serum albumin. Cells were incubated with anti-cathepsin $\mathrm{D}$ antibody overnight at $4{ }^{\circ} \mathrm{C}$ and then incubated with TRITC-labeled secondary antibody at room temperature for $2 \mathrm{~h}$. Nuclei were stained with 4',6-diamidino-2-phenylindole (DAPI). Cells were visualized by Z-Stack imaging with a confocal microscope (Olympus FV1000; Tokyo, Japan) and processed using Fluoview software (Version 2.0, Olympus).

\subsection{In Vitro Measurement of Membrane Permeabilization}

Large unilamellar vesicles (LUVs) mimicking the composition of lysosomal membranes were used as model systems to investigate the impact of ESeroS-GS on membrane permeabilization [15]. In brief, DOPC (70\%), DOPE (20\%) and DOPA (10\%) were mixed from stocks dissolved in chloroform. The organic solvent was removed by evaporation under a stream of nitrogen gas, followed by incubation for $2 \mathrm{~h}$ in a vacuum to ensure complete solvent removal. Lipid films were resuspended in HEPES buffer (10 mM HEPES, pH 7.4, $50 \mathrm{mM} \mathrm{NaCl}, 0.2 \mathrm{mM}$ EDTA), and subjected to 10 freeze-thaw cycles. Large unilamellar vesicles (LUVs) were then formed by extrusion through 100-nm nucleopore polycarbonate membranes.

The permeabilization of membrane was determined by monitoring the fluorescence of 5-carboxyfluorescein (CF)-containing liposomes. To prepare CF-containing liposomes, $50 \mathrm{mM} \mathrm{CF}$ was dissolved in the HEPES buffer, and LUVs were prepared with the CF-containing HEPES buffer. Non-entrapped fluorophores were removed by centrifugation at 45,000 rpm for 30 min with a Beckman TL-100.3 ultracentrifuge (Beckman, Brea, CA, USA). The leakage assay was performed as described previously [31]. Following incubation of tBid with LUVs (phospholipids concentration $100 \mu \mathrm{M}$ ) with or without EseroS-GS in $100 \mu \mathrm{L}$ of HEPES buffer at $30^{\circ} \mathrm{C}$ for $30 \mathrm{~min}$, the release of CF was quantified using a Hitachi F-4500 flurospectrometer (Hitachi, Tokyo, Japan) with $5 \mathrm{~nm}$ bandwidths centered at 493 and $513 \mathrm{~nm}$ for excitation and emission, respectively (R). The control followed the same procedure except for the addition of buffer instead of protein (B). Addition of Triton X-100 to a final concentration of $1 \%(v / v)$ was used as a positive control for $100 \%$ CF release $(\mathrm{T})$. The percentage of leakage was determined by:

$$
\text { Leakage }(\%)=[(\mathrm{R}-\mathrm{B}) /(\mathrm{T}-\mathrm{B})] \times 100
$$

\subsection{Binding of tBid to Lysosomal and Model Membranes}

Lysosomes were purified from rat cerebral cortex and were suspended in buffer containing $250 \mathrm{mM}$ sucrose, $20 \mathrm{mM}$ HEPES, pH 7.2 [26]. Then, lysosomes were incubated with tBid with or without EseroS-GS for $30 \mathrm{~min}$, respectively, followed by centrifugation at $18,000 \times g$ for $10 \mathrm{~min}$. The pellets (lysosomes) were subjected to immunoblot analysis to detect the binding of tBid to lysosomal membranes.

For detection of tBid bound to model membranes, LUVs prepared from DOPC (70\%), DOPE $(20 \%)$ and DOPA $(10 \%)$ was incubated with tBid with or without ESeroS-GS in $100 \mu \mathrm{L}$ of HEPES buffer at $30{ }^{\circ} \mathrm{C}$ for $30 \mathrm{~min}$. Then, the samples were centrifuged at 45,000 rpm for $30 \mathrm{~min}$. The pellets were washed three times with the HEPES buffer and then were subjected to immunoblot analysis to detect the binding of tBid to LUV membranes.

The insertion of tBid into the LUV bilayer membranes was also analyzed by measuring the fluorescence of PM-labeled tBid. Briefly, tBid was labeled with PM to form PM-labeled tBid and then incubated with LUVs prepared from DOPC (70\%), DOPE (20\%) and DOPA (10\%) in HEPES at $30{ }^{\circ} \mathrm{C}$ for $30 \mathrm{~min}$. Fluorescence measurements were performed with a Hitachi F-4500 fluorescence spectrometer set to $340 \mathrm{~nm}$ excitation and $376 \mathrm{~nm}$ emission with $5 \mathrm{~nm}$ slit widths.

\subsection{Assay of tBid Oligomerization}

The oligomerization of $\mathrm{tBid}$ was measured by a cross-linking assay. tBid was incubated with LUVs prepared from DOPC (70\%), DOPE (20\%) and DOPA (10\%) in buffer containing $10 \mathrm{mM} \mathrm{HEPES}$ (PH 7.4), $0.1 \mathrm{M} \mathrm{NaCl}$ at $37^{\circ} \mathrm{C}$ for $30 \mathrm{~min}$ and then the samples were centrifuged at $45,000 \mathrm{rpm}$ for 
$30 \mathrm{~min}$. Sulfo-BSOCOES (in dimethyl sulfoxide) was added to the pellets from at a final concentration of $10 \mathrm{mM}$. After incubation for $2 \mathrm{~h}$ at room temperature, the cross-linker was quenched by the addition of $1 \mathrm{M}$ Tris- $\mathrm{HCl}(\mathrm{pH} 7.5)$ to a final concentration of $20 \mathrm{mM}$. Then, the samples were lysed and subjected to SDS-PAGE. Immunoblot analysis was performed to analyze the oligomerization of $\mathrm{tBid}$.

\subsection{Immunoblot Analysis}

Samples were resolved by 15\% SDS-PAGE, transferred to polyvinylidene fluoride membranes, and probed with proper primary antibodies. The membranes were incubated with peroxidase-conjugated secondary antibodies and visualized using a Amersham Pharmacia ECL chemiluminescent kit (GE, Marlborough, MA, USA) and Kodak X-OMAT films (Rochester, NY, USA).

\subsection{Data Analysis}

All data were expressed as the mean \pm SD unless otherwise indicated. Differences between groups were compared by analysis of variance followed by post hoc Bonferroni tests to correct for multiple comparisons. Differences were considered to be statistically significant at $p<0.05\left({ }^{*}\right)$ or $\left.p<0.01{ }^{* *}\right)$.

\section{Conclusions}

We reported here that ESeroS-GS effectively scavenged free radicals and protected neuronal cells from oxidative stress-induced cell death by stabilizing lysosomes. Oxidative stress induced lysosomal membrane permeabilization rapidly and caused the redistribution of lysosomal proteases, which was responsible for the neuronal cell death. ESeroS-GS abolished the interaction between $\mathrm{tBid}$ and the lysosomal membranes, blocked the translocation of $\mathrm{tBid}$ to the lysosomal membranes, decreased its oligomerization within the membrane circumstances, prevented the lysosomal membrane permeabilization, and thus attenuated the neuronal cell death. These data suggest that ESeroS-GS protected the neuronal cells from oxidative stress by stabilizing lysosomal membranes, and thus might act as a novel neuroprotector for neuronal diseases associated with oxidative stress. However, other mechanisms, such as the induction and activation of Nrf2/Keap1/ARE pathway, may also be involved in the neuroprotection by ESeroS-GS. We will focus on this key pathway in future research.

Acknowledgments: The authors thank Kazumi Ogata (Senju Pharmaceutical Co. Ltd., Osaka, Japan) for providing the ESeroS-GS and Akitane Mori (School of Medicine, Okayama Unviersity, Japan) for valuable suggestions and discussions. The authors are also indebted to Wenmin Zhong (Institute of Biophysics, Chinese Academy of Sciences, Beijing, China) for technical assistance. This work was supported by grants from the National Basic Research Program of China (Grant 2012CB934003), the National Natural Science Foundation of China (31300683 and 31470814), and the Major Equipment Program of China (Grant 2011YQ030134).

Author Contributions: Taotao Wei conceived and designed the experiments; Xingyu Zhao performed the free radical scavenging experiments; Na Yang and Qianqian Chen performed cell culture experiments; Na Yang and Xiaolong He purified the recombinant proteins and performed related experiments. Taotao Wei coordinated writing the paper to which all co-authors contributed.

Conflicts of Interest: The authors declare no conflict of interest.

\section{Abbreviations}

The following abbreviations are used in this manuscript:

$\begin{array}{ll} & \gamma \text { - L-glutamyl-S-[2-[[[3,4-dihydro-2,5,7,8-tetramethyl- } \\ \text { EseroS-GS } & \text { 2-(4,8,12-trimethyltridecyl)-2H-1-benzopyran-6-yl]oxy]carbonyl]- } \\ & \text { 3-[[2-(1H-indol-3-yl)ethyl]amino]-3-oxopropyl]-L-cysteinylglycine sodium salt } \\ \text { ROS } & \text { reactive oxygen species } \\ \text { LMP } & \text { lysosomal membrane permeabilization } \\ \text { Bid } & \text { BH3 interacting domain death agonist } \\ \text { tBid } & \text { truncated Bid } \\ \text { ABTS } & \text { cation radical of 2,2'-azinobis(3-ethylbenzothiazoline)-6-sulfonate } \\ \text { DPPH } & \text { 2,2-diphenyl-1-picrylhydrazyl free radical } \\ \text { ESR } & \text { Electron Spin Resonance }\end{array}$




$\begin{array}{ll}\text { Trolox } & \text { 6-hydroxy-2,5,7,8-tetramethychroman-2-carboxylic acid } \\ \text { MTT } & \text { 3-(4,5-dimethyl-2-thiazolyl)-2,5-diphenyl-2-H-tetrazolium bromide } \\ \text { AO } & \text { acridine orange } \\ \text { TPCK } & \text { N-p-tosyl-L-phenylalanine chloromethyl ketone } \\ \text { E64d } & \text { (2S,3S)-trans-epoxysuccinyl-L-leucylamido-3-methylbutane ethyl ester } \\ \text { LUV } & \text { large unilamellar vesicle } \\ \text { PM } & \text { N-(1-pyrenyl)maleimide } \\ \text { DOPA } & \text { 1,2-dioleoyl-sn-glycero-3-phosphate } \\ \text { DOPC } & \text { 1,2-dioleoyl-sn-glycero-3-phosphocholine } \\ \text { DOPE } & \text { 1,2-dioleoyl-sn-glycero-3-phosphoethanolamine } \\ \text { HRP } & \text { horseradish peroxidase } \\ \text { TRITC } & \text { 6-tetramethylrhodamine isothiocyanate } \\ \text { CF } & \text { 5-carboxyfluorescein } \\ \text { sulfo-BSOCOES } & \text { bis[2-(sulfosuccinimidooxycarbonyloxy)ethyl] sulfone } \\ \text { EDTA } & \text { ethylenediaminetetraacetic acid disodium salt }\end{array}$

\section{References}

1. Zorov, D.B.; Juhaszova, M.; Sollott, S.J. Mitochondrial reactive oxygen species (ROS) and ROS-induced ROS release. Physiol. Rev. 2014, 94, 909-950. [CrossRef] [PubMed]

2. Santilli, F.; D'Ardes, D.; Davi, G. Oxidative stress in chronic vascular disease: From prediction to prevention. Vascul. Pharmacol. 2015, 74, 23-37. [CrossRef] [PubMed]

3. Tong, L.; Chuang, C.C.; Wu, S.; Zuo, L. Reactive oxygen species in redox cancer therapy. Cancer Lett. 2015, 367, 18-25. [CrossRef] [PubMed]

4. Jha, J.C.; Banal, C.; Chow, B.S.; Cooper, M.E.; Jandeleit-Dahm, K.A. Diabetes and kidney disease: Role of oxidative stress. Antioxid. Redox. Signal. 2016. [CrossRef] [PubMed]

5. Cobb, C.A.; Cole, M.P. Oxidative and nitrative stress in neurodegeneration. Neurobiol. Dis. 2015, 84, 4-21. [CrossRef] [PubMed]

6. Espinet, C.; Gonzalo, H.; Fleitas, C.; Menal, M.J.; Egea, J. Oxidative stress and neurodegenerative diseases: A neurotrophic approach. Curr. Drug Targets 2015, 16, 20-30. [CrossRef] [PubMed]

7. Bhola, P.D.; Letai, A. Mitochondria-judges and executioners of cell death sentences. Mol. Cell. 2016, 61, 695-704. [CrossRef] [PubMed]

8. Green, D.R. Apoptotic pathways: The roads to ruin. Cell. 1998, 94, 695-698. [CrossRef]

9. Ricci, J.E.; Waterhouse, N.; Green, D.R. Mitochondrial functions during cell death, a complex (I-V) dilemma. Cell. Death Differ. 2003, 10, 488-492. [CrossRef] [PubMed]

10. Green, D.R. Apoptotic pathways: ten minutes to dead. Cell. 2005, 121, 671-674. [CrossRef] [PubMed]

11. Ferri, K.F.; Kroemer, G. Organelle-specific initiation of cell death pathways. Nat. Cell Biol. 2001, 3, E255-E263. [CrossRef] [PubMed]

12. Repnik, U.; Stoka, V.; Turk, V.; Turk, B. Lysosomes and lysosomal cathepsins in cell death. Biochim. Biophys. Acta 2012, 1824, 22-33. [CrossRef] [PubMed]

13. Terman, A.; Kurz, T. Lysosomal iron, iron chelation, and cell death. Antioxid. Redox Signal. 2013, 18, 888-898. [CrossRef] [PubMed]

14. Zhao, K.; Zhao, X.; Tu, Y.; Miao, Q.; Cao, D.; Duan, W.; Sun, Y.; Wang, J.; Wei, T.; Yang, F. Lysosomal chymotrypsin B potentiates apoptosis via cleavage of Bid. Cell Mol. Life Sci. 2010, 67, 2665-2678. [CrossRef] [PubMed]

15. Zhao, K.; Zhou, H.; Zhao, X.; Wolff, D.W.; Tu, Y.; Liu, H.; Wei, T.; Yang, F. Phosphatidic acid mediates the targeting of tBid to induce lysosomal membrane permeabilization and apoptosis. J. Lipid Res. 2012, 53, 2102-2114. [CrossRef] [PubMed]

16. Kelsey, N.A.; Wilkins, H.M.; Linseman, D.A. Nutraceutical antioxidants as novel neuroprotective agents. Molecules 2010, 15, 7792-7814. [CrossRef] [PubMed]

17. Iriti, M.; Vitalini, S.; Fico, G.; Faoro, F. Neuroprotective herbs and foods from different traditional medicines and diets. Molecules 2010, 15, 3517-3555. [CrossRef] [PubMed]

18. Wei, T.; Zhao, X.; Hou, J.; Ogata, K.; Sakaue, T.; Mori, A.; Xin, W. The antioxidant ESeroS-GS inhibits NO production and prevents oxidative stress in astrocytes. Biochem. Pharmacol. 2003, 66, 83-91. [CrossRef] 
19. Duan, W.; Zhou, J.; Zhang, S.; Zhao, K.; Zhao, L.; Ogata, K.; Sakaue, T.; Mori, A.; Wei, T. ESeroS-GS modulates lipopolysaccharide-induced macrophage activation by impairing the assembly of TLR-4 complexes in lipid rafts. Biochim. Biophys. Acta 2011, 1813, 772-783. [CrossRef] [PubMed]

20. Zhao, L.; Zhao, X.; Zhao, K.; Wei, P.; Fang, Y.; Zhang, F.; Zhang, B.; Ogata, K.; Mori, A.; Wei, T. The $\alpha$-tocopherol derivative ESeroS-GS induces cell death and inhibits cell motility of breast cancer cells through the regulation of energy metabolism. Eur. J. Pharmacol. 2014, 745, 98-107. [CrossRef] [PubMed]

21. Re, R.; Pellegrini, N.; Proteggente, A.; Pannala, A.; Yang, M.; Rice-Evans, C. Antioxidant activity applying an improved ABTS radical cation decolorization assay. Free Radic. Biol. Med. 1999, 26, 1231-1237. [CrossRef]

22. Csepregi, K.; Neugart, S.; Schreiner, M.; Hideg, E. Comparative evaluation of total antioxidant capacities of plant polyphenols. Molecules 2016, 21, 208. [CrossRef] [PubMed]

23. Kim, Y.K.; Guo, Q.; Packer, L. Free radical scavenging activity of red ginseng aqueous extracts. Toxicology 2002, 172, 149-156. [CrossRef]

24. Kotali, A.; Nasiopoulou, D.A.; Tsoleridis, C.A.; Harris, P.A.; Kontogiorgis, C.A.; Hadjipavlou-Litina, D.J. Antioxidant activity of 3-[N-(acylhydrazono)ethyl]-4-hydroxy-coumarins. Molecules 2016, 21, 138. [CrossRef] [PubMed]

25. Kurz, T.; Terman, A.; Gustafsson, B.; Brunk, U.T. Lysosomes and oxidative stress in aging and apoptosis. Biochim. Biophys. Acta 2008, 1780, 1291-1303. [CrossRef] [PubMed]

26. Miao, Q.; Sun, Y.; Wei, T.; Zhao, X.; Zhao, K.; Yan, L.; Zhang, X.; Shu, H.; Yang, F. Chymotrypsin B cached in rat liver lysosomes and involved in apoptotic regulation through a mitochondrial pathway. J. Biol. Chem. 2008, 283, 8218-8228. [CrossRef] [PubMed]

27. Zhai, D.; Huang, X.; Han, X.; Yang, F. Characterization of tBid-induced cytochrome c release from mitochondria and liposomes. FEBS Lett. 2000, 472, 293-296. [CrossRef]

28. Resink, A.; Hack, N.; Boer, G.J.; Balazs, R. Growth conditions differentially modulate the vulnerability of developing cerebellar granule cells to excitatory amino acids. Brain Res. 1994, 655, 222-232. [CrossRef]

29. Arthur, P.G.; Niu, X.; Rigby, P.; Steer, J.H.; Jeffrey, G.P. Oxidative stress causes a decline in lysosomal integrity during hypothermic incubation of rat hepatocytes. Free Radic. Biol. Med. 2008, 44, 24-33. [CrossRef] [PubMed]

30. Doulias, P.T.; Kotoglou, P.; Tenopoulou, M.; Keramisanou, D.; Tzavaras, T.; Brunk, U.; Galaris, D.; Angelidis, C. Involvement of heat shock protein-70 in the mechanism of hydrogen peroxide-induced DNA damage: the role of lysosomes and iron. Free Radic. Biol. Med. 2007, 42, 567-577. [CrossRef] [PubMed]

31. Yan, L.; Miao, Q.; Sun, Y.; Yang, F. tBid forms a pore in the liposome membrane. FEBS Lett. 2003, 555, 545-550. [CrossRef]

Sample Availability: Samples of the compound EseroS-GS is available from the authors.

(C) 2016 by the authors; licensee MDPI, Basel, Switzerland. This article is an open access article distributed under the terms and conditions of the Creative Commons Attribution (CC-BY) license (http://creativecommons.org/licenses/by/4.0/). 